\title{
Considerations in pre-operative assessment of rheumatoid patients
}

\begin{abstract}
Rheumatoid disease is common amongst surgical patients and it is vitally important that modifiable risk factors are addressed preoperatively. The aim of this review article is to look at the common risk factors associated with rheumatoid patients receiving surgery and offer recommendations on what steps should be taken preoperatively to minimize these. The currently literature was reviewed and a list of recommendation was given using best current evidence.
\end{abstract}

Keywords: Rheumatoid, Pre-operative, Assessment
Volume I Issue I - 2014

\author{
Stephanie Spence,' Bilal Jamal, ${ }^{2}$ Anand Pillai ${ }^{3}$ \\ 'Department of Trauma and Orthopaedics, Western Infirmary \\ Glasgow, UK \\ ${ }^{2}$ Department of Trauma and Orthopaedics,Victoria Infirmary \\ Glasgow, UK \\ ${ }^{3}$ Department of Trauma and Orthopaedics, University Hospitals \\ of South Manchester, UK
}

\begin{abstract}
Correspondence: Bilal Jamal, Department of Trauma and Orthopaedics, University Hospitals of South Manchester, 88 Earlspark Avenue, Newlands, Glasgow, UK, Tel 07584-169-123, Emailbjamal@doctors.org.uk
\end{abstract}

Received: May 26, 2014 | Published: July 05, 2014

\section{Introduction}

Rheumatoid arthritis is an inflammatory disease of unknown etiology. ${ }^{1}$ The world wide prevalence is $1 \%$ and the incidence in the UK population per 100000/year is 1.5 in men and 3.6 in women. ${ }^{2}$ The disease varies greatly in its severity and rate of progression and affects patients of all ages. ${ }^{3}$ Surgical procedure amongst this group of patients are common with $>25 \%$ having an orthopaedic procedure 10-20 years after being diagnosed and further more due to the associated systemic complications they also commonly require other subspecialty surgeries. ${ }^{4}$ It is important to recognize that, in a surgical setting, these patients have specific potential risks factors that need to be addressed and they should not be treated like the routine preoperative candidate.

It is widely reported in the literature that these patients pose a much higher risk of postoperative infection and thromboembolic events. The perioperative period is also much higher risk due to an association with cardiovascular disease as well as instability of the cervical spine that can compromise the integrity of the spinal cord and may result in a myelopathy or in rare cases sudden death. ${ }^{5}$ This article aims to address these issues and suggest a preoperative management pathway to ensure rheumatoid patients undergoing a surgical procedure are optimally managed thus giving them the best possible outcome for their chosen procedure.

\section{Discussion}

The initial assessment of the suitability of these patients for an anesthetic and surgery should begin with taking a routine history and performing a full examination. It is important to document how well controlled their disease is, any previous procedures, an up to date medication list including current disease modifying anti-rheumatic drugs (DMARDs) and associated features of rheumatoid disease. During their physical examination you must assess their cardio respiratory status to guide further investigations. It is also important to note musculoskeletal involvement, which will help in the planning of positioning on the table for their procedure. Potential difficulties with anaesthetizing rheumatoid patients are discussed in detail below and summarized in Table 1.4

Table 1 Potential difficulties with anaesthetizing rheumatoid patients

\begin{tabular}{ll}
\hline I & Anesthetic concerns in RA patients \\
2 & Increased cardiovascular risk \\
3 & Likely pre-existing anemia \\
4 & Fragile Skin \\
5 & Deformity and rigidity of joints \\
6 & Extreme pain in joints \\
7 & Poor peripheral venous access \\
8 & Difficult airway management \\
9 & Glucose replacement if on long term steroids \\
I0 & High spinal blocks \\
II & Risk of perioperative neurological damage \\
I2 & Extended post op intubation if Myopathy \\
\hline
\end{tabular}

\section{Cervical spine}

So far there remains no consensus on the role of plain $\mathrm{x}$-rays in the evaluation of instability of the cervical spine in rheumatoid patients. Between $17-86 \%$ of patients will have evidence of cervical spine disease 5 years after diagnosis. The main concern is an iatrogenic spinal cord injury during the positioning of the head and neck during the intubation phase of the procedure. To overcome this any patient with a spine classed as unstable the anesthetist may perform an awake fiber-optic intubation in place of the traditional intubation. Here the patient receives local anesthetic to their airway and the intubation tube is placed with the neck in a neutral position followed by motor neurological testing to ensure no damage has occurred. Inductions with general anesthetic can then follow. ${ }^{4}$ 
The difficulty presents when deciding upon which patients have an unstable cervical spine and does performing preoperative radiographs help in this decision. In patients who are symptomatic with neck pain or neurological deficit then it is recommended to proceed with preoperative investigation in the form of flexion and extension radiographs/MRI scans. ${ }^{4}$ It is not so clear who should be investigated in the asymptomatic group. There are multiple reports in the literature that state that flexion and extension views are more sensitive than normal neutral views (which can miss up to $50 \%$ of cases) and pick up $44 \%$ of asymptomatic cervical spine instability. ${ }^{5,6}$ It is also reported that patients presenting for an orthopaedic procedure are at a higher risk of instability than those presenting for general surgery procedures as by the time they require orthopaedic intervention they are more likely to have failed medical management and there is a greater chance of multiple joints being more severely affected. ${ }^{5}$ Another report by Wasserman et al. ${ }^{7}$ also recommends when screening radiographs show spinal canal space is $<13 \mathrm{~mm}$ further imaging in the form of an MRI should be performed and consideration of discussion with a spinal surgeon prior to elective surgery should be made.

Despite the above evidence a recent paper by Lopez-Olivo et al. ${ }^{4}$ looking solely at the use of pre-operative radiographs in rheumatoid patients concluded that the decision about intubation technique lies with individual anesthetists and regardless of the radiographic findings, cervical spine stabilization and modification of intubation technique was not always performed. This was thought to be due to experience, skill and time factors involved with awake fiber-optic intubation and many anesthetists felt more comfortable with more traditional methods. Although there has been reported neurological deficit in $10-36 \%$ of patients with cervical spine pathology following surgery there was not cases in this study. They also highlighted that less than $1 / 3$ of radiographs performed were deemed to be adequate.

\section{Cardiovascular risk factors}

It has been well reported that rheumatoid patients have a higher risk of cardiovascular disease. Often they do not present with classical symptoms and the presence of this disease can be missed. ${ }^{4}$ Rheumatoid disease has been shown to have a similar level of risk of cardiac disease in patients as diabetes. ${ }^{8}$ There are several mechanisms that are thought to contribute to this although the exact reason remains uncertain. ${ }^{4}$ Firstly, although they rarely present with angina rheumatoid patients have a much higher risk of a myocardia infarction and they are six times more likely to suffer from sudden death following this than the general population. Another common presentation of cardiac disease in rheumatoid patients is heart failure. They often present with no history of ischemic heart disease and they are twice as likely to develop this compared with the general population. ${ }^{4}$

It is also reported that cardiac arrhythmias are a cause of mortality in these patients. Tachyarrhythmias are poorly tolerated as they often have systolic dysfunction and there are frequently conduction abnormalities from rheumatoid nodules and amyloidosis. ${ }^{9}$ With this in mind preoperative assessment of cardiac function must routinely include an electrocardiogram to assess left ventricular dysfunction and conduction abnormalities. An echocardiogram should also be performed. It can be falsely reassuring if the ejection fraction is reported as normal so it is important to ask the technician to look specifically for left ventricular hypertrophy, valvular abnormalities and diastolic dysfunction. ${ }^{4}$

It is also worth noting that anemia in these patients is common due to anemia of chronic disease as well as a side effect from certain drug therapies and they frequently require perioperative blood transfusion. There should also remain a low threshold for ordering respiratory investigations as there are associated extra-articular manifestations that involve the lungs. Investigations to be considered are a chest radiograph, lung function tests and arterial gases.

\section{Infection}

Patients with rheumatoid disease are known to be at a higher risk of infection and when the disease is active this increases risk furthermore. ${ }^{10}$ In the case of orthopaedic patients this is also compounded by the fact that surgery is technically more difficult, requiring a longer operative time and it has been shown that rheumatoid patients have a 1.6 greater chance of having an arthroplasty revision for infection compared with osteoarthritis patients. ${ }^{5}$

Another factor associated with these patients is the medications they are commonly on and the well known immunosuppressive effects of these. In particular biological and synthetic DMARDs and corticosteroids are becoming more common practice in the management of rheumatoid patients. This is a topical matter as the benefits of these drugs are widely reported in the literature however the perioperative risks of these are not well defined. It is important to consider the drug management pre operatively on a patient-to-patient basis and weigh up the increased risk of post-operative infection when on DMARDs with the increased risk of disease flare up and progression when DMARDs are withheld. The most commonly reported DMARD in the literature is Methotrexate. A study by Grennan et al. ${ }^{11}$ randomized 388 orthopaedic patients with rheumatoid arthritis receiving surgery into two groups: one to withhold their Methotrexate and the other to continue it during the perioperative period. They also compared this with a control group of 228 patients with rheumatoid arthritis not on Methotrexate. They found that the group who had the least amount of postoperative infections, wound complications and flare of disease was the group who continued Methotrexate. It has since been recommended that Methotrexate should continue when having a surgical procedure.

Another commonly used drug group in rheumatoid disease is corticosteroids as they are effective in symptom control as well as exhibiting mild DMARD properties. It is well reported that infection risk increases alongside dose and duration of these drugs. There is also an associate risk of adrenal insufficiency and hemodynamic collapse so preoperative planning is essential, as these patients will require perioperative steroid administration, although it has been shown that preoperative adrenal testing is not beneficial. ${ }^{5}$ Patients who are corticosteroid therapy for adrenal insufficiency should have increased doses of steroids perioperatively where as those on it for disease control should simply have their usual dose on the morning of surgery. ${ }^{12}$

Finally, with the advent of biological agents including TNF alpha the incidence of infection has increased but due to the excellent symptomatic control, and in some case remission of disease, this has been accepted. There have been several studies that have shown an increased post-operative wound infection rates and these have provided the basis for guidelines published by both American Rheumatology Association and the British Society of Rheumatology. ${ }^{13}$ They recommend that biological drugs should be stopped 1-4 weeks prior to surgery and restarted 1-4 weeks after surgery depending on wound healing. It is also worth considering the combined effects of 
these drugs alongside other groups such as corticosteroids and patient comorbidities like diabetes.

\section{Thromboembolic events}

Evidence of a link between rheumatoid disease and increased thromboembolic events is not reported widely in the literature. The exact perioperative risk is still unknown. ${ }^{5}$ However patients with rheumatoid disease are thought to be in a hypercoagulable state due to chronic inflammation as well as often being on corticosteroids preoperatively. ${ }^{4,5}$ They also frequently have multiple joint involvements and may be slower to mobilize and progress postoperatively increases their stay in hospital. A previous retrospective review looking at 609 rheumatoid cases showed that there was an increase in VTE events compared with the general population and patients with severe, extra-articular disease had a significantly increased risk of a VTE. ${ }^{14}$ However it is also well established that hospitalization increases your VTE risk and there has been no evidence to show that this rate is greater in the rheumatoid group over the general population. ${ }^{5}$ The consensus seems to be that for operative procedures, especially in orthopaedic lower limb surgeries, patients should be treated as high risk like any other patient due hospitalization and the surgery itself and there is no evidence to suggest they require a different protocol for DVT prophylaxis.

\section{Conclusion}

In conclusion it is vitally important that rheumatoid patients are fully assessed in the preoperative setting to ensure all modifiable risks can be kept to a minimum. From the evidence described in this paper our authors would recommend the following considerations.

1. In cervical spine asymptomatic patients there is still controversy surrounding the need for further investigation. However, our authors would recommend that flexion and extension views should be routinely obtained prior to these patients receiving surgery.

2. Patients should also receive flexion and extension views of their lumbar spine routinely to aid with their positioning on the surgical table.

3. If patients present with symptoms of instability, either clinically or radio graphically, they should received imaging of their spine preoperatively in the form of MRI or CT scanning in addition to their flexion and extension radiographs. Depending on these results a spinal surgical option may be sought.

4. All patients should receive an ECG and an echocardiogram preoperatively as these patients often have asymptomatic cardiac disease.

5. Aroutine haemoglobin analysis should be performed preoperatively and crossed matched blood should be available preoperatively for patients receiving major surgery.

6. In all patients, regardless of their history of respiratory manifestations of rheumatoid disease, a chest radiograph should be performed as well as pulmonary function tests and arterial gases especially if they describe a poor exercise function.

7. Methotrexate should be continued throughout the surgical process.
8. Corticosteroids should be given at their normal dose on the morning of surgery unless they are indicated for adrenal insufficiency in which case perioperative steroid treatment will be required.

9. Biological agents should be stopped 1-4 weeks prior to surgery and recommenced 1-4 weeks post operatively depending on wound healing.

10. In the surgical setting rheumatoid patients have not been proven to have an increased risk of VTE and should be treated with the standard local protocol for DVT prophylaxis.

11. Finally, if the pre surgical assessment identifies that a patient is particularly high risk for surgery then there should be serious consideration of whether the patient is a candidate for an elective procedure at all.

It is important to involve the anesthetic team early on when managing these patients preoperatively. If in doubt about specific DMARDs rheumatological advice should be sought.

\section{Acknowledgment}

None.

\section{Conflicts of interest}

This is to confirm that there are no conflicts of interest to declare for this work. No financial or other inducements have been received or are expected as a result of this paper being written.

\section{References}

1. Kelley JT, Conn DL. Perioperative management of the rheumatic disease patient. Bull Rheum Dis. 2002;51(6)

2. Samanta R, Shoukrey K, Griffiths R. Rheumatoid arthritis and anaesthesia Anaesthesia. 2011;66(12):1146-1159.

3. Axford JS. Preoperative evaluation and perioperative management of patients with rheumatic disease. Up To Date, 2014.

4. Lopez-Olivo MA, Andrabi TR, Palla SL, et al. Cerical Spine Radiographs in Patients with rheumatoid arthritis undergoing anaethesia. J Clin Rheumatol. 2012;18(2):61-66.

5. Goodman SM. Rheumatoid Arthritis: Preoperative Evaluation for Total Hip and Total Knee Replacement Surgery. J Clin Rheumatol. 2013;19(4):187192.

6. Kauppi M, Neva MH. Sensitivity of lateral view cervical spine radiographs taken in the neutral position in atlantoaxial subluxation in rheumatic diseases. Clin Rheumatol. 1998;17(6):511-514.

7. Wasserman BR, Moskovich R, Razi AE. Rheumatoid arthritis of the cervical spine-clinical considerations. Bull NYU Hosp Jt Dis. 2011;69(2):136-148.

8. Peters MJ, van Halm VF, Voskuyl AE, et al. Does rheumatoid arthritis equal diabetes mellitus as an independent risk factor for cardiovascular disease? A prospective study. Arthritis and Rheum. 2009;61(11):1571-1579.

9. Gabriel SE. Why do people with rheumatoid arthritis still die prematurely? Ann Rheum Dis. 2008;67(3):30-34.

10. Au K, Reed G, Curtis JR, et al. High disease activity is associated with an increased risk of infection in patients with rheumatoid arthritis. Ann Rheum Dis. 2011;70(5):785-791. 
11. Grennan DM, Gray J, Loudon J, et al. Methotrexate and early postoperative complications in patients with rheumatoid arthritis undergoing elective orthopaedic surgery. Ann Rheum Dis. 2001;60(3):214-217.

12. Marik PE, Varon J. Requirement of perioperative stress doses of corticosteroids: a systematic review of the literature. Arch Surg. 2008;143(12):1222-1226.
13. Ding $\mathrm{T}$, Ledingham J, Luqmani $\mathrm{R}$, et al. BSR and BHPR rheumatoid arthritis guidelines on safety of anti-TNF therapies. Rheumatology (Oxford). 2010;49(11):2217-2219.

14. McEntegart A, Capell HA, Creran D, et al. Cardiovascular risk factors, including thrombotic variables, in a population with rheumatoid arthritis. Rheumatology (Oxford). 2001;40(6):640-644. 